Escaneado con CamScanner 


\title{
Análisis de las causas de errores de sentido en traducciones literarias alemán-español
}

\author{
$M^{\prime \prime}$ Pilar Castillo Bernal \\ Universidad de Córdoba \\ z92cabep@uco.es \\ https://dx.doi.org/10.12795/futhark.2014.i9.02
}

\begin{abstract}
This paper discusses the analysis of errors of meaning in translations made by students of Literary Translation C $>$ A GermanSpanish. This subject was formerly taught in the fourth year of the Degree in Translation and Interpreting at the University of Cordoba. The contents analysed are fragments of literary works in German language translated into Spanish by students in the course of the ongoing assessment process. The tests comprise two different genres: drama and novel. The purpose is first to identify the gaps in students' performance regarding language and translation competences, in order to take corrective measures in the classroom. The second purpose is to establish a grading system to be consistently applied in the evaluation of tests. Thirdly, this analysis aims at providing feedback for syllabus planning of this subject in the B.A. Translation and Interpreting of the University of Cordoba. As to the findings, the main language gaps identified include mainly the lack of mastery of grammar structures and literary features of the corresponding works. Similarly, the need for a flexible grading system was detected in order to assess the impact of a given error on each translation. Fragments or terms that are considered particularly complex should be given extra points when properly translated.
\end{abstract}

Key words: literary translation, didactics, grading system, errors of meaning. 
Resumen: En la presente comunicación se expondrá el análisis de los errores de sentido detectados en traducciones realizadas por el alumnado de la asignatura Traducción Literaria $\mathrm{C}>\mathrm{A}$ alemán-español, impartida en cuarto curso en la Licenciatura en Traducción e Interpretación de la Universidad de Córdoba. Los contenidos analizados son fragmentos de obras literarias en lengua alemana traducidas al español por el alumnado en el marco de dos pruebas parciales en el marco de la evaluación continua, correspondientes a géneros textuales diversos como son el drama y la novela. Los objetivos son, en primer lugar, determinar las carencias del alumnado en cuanto a destrezas lingüísticas y competencias traductológicas, de cara a tomar las oportunas medidas correctivas que ayuden a mejorar el proceso de enseñanza-aprendizaje. En segundo lugar, establecer un sistema de puntuación de dichos errores para su uso consistente en la evaluación de las pruebas. En tercer lugar, este análisis aspira a servir de retroalimentación para la programación de la asignatura de Traducción Literaria de la lengua $C$ (alemán), impartida en el tercer curso en el Grado en Traducción e Interpretación de la Universidad de Córdoba. En cuanto a las conclusiones del trabajo, las carencias lingǘsticas identificadas consistían principalmente en la falta de familiarización del alumnado con las estructuras gramaticales y recursos literarios propios del lenguaje de las obras en cuestión. Asimismo, se detectó la necesidad de un sistema de puntuación flexible y adaptado a las implicaciones de cada error en cada traducción, con bonificación de los fragmentos o términos que pudieran ser considerados dificultades de traducción, en caso de su correcta resolución.

Palabras clave: traducción literaria, didáctica, puntuación, errores de sentido.

\section{INTRODUCCIÓN}

Los estudios de Traducción e Interpretación, al igual que la Traductología, son disciplinas relativamente jóvenes de reciente implantación en nuestro país. Las primeras Escuelas Universitarias de Traductores e Intérpretes se crearon en Barcelona (la por entonces llamada Escuela Universitaria de Idiomas, según Decreto de 18 de agosto de 1972) y Granada (Real Decreto 2572/1979, de 14 de septiembre de 1979). En ellas se cursaban estudios de tres años para obtener la Diplomatura en Traducción e Interpretación. 
Desde entonces, los planes de estudio han sido modificados en dos ocasiones, por primera vez en 1991 al convertirse en estudios de licenciatura (BOE 30-9-91) y posteriormente en 2001 (BOE 14-04-01). Actualmente nos encontramos en fase de transición de la licenciatura al grado en traducción e interpretación, tal como recoge el Libro Blanco del Título de Grado en Traducción e Interpretación de la ANECA. ${ }^{1}$

A pesar de su corta historia, los estudios de traducción e interpretación han experimentado un vertiginoso ascenso en las últimas décadas. ${ }^{2}$ Ante esta verdadera expansión las facultades, especialmente las de reciente creación, se enfrentan a un doble desafío: por una parte, perfilarse como centros destacados en la formación de traductores e intérpretes frente a la creciente competencia, y por otro el paso de la licenciatura al grado, cumpliendo con las nuevas directrices del Espacio Europeo de Educación Superior creado a partir de la firma de la Declaración de Bolonia en 1999. En este sentido, la implantación de los nuevos planes de estudio supone una oportunidad extraordinaria para evaluar y optimizar los enfoques didácticos y metodológicos.

Es evidente que la consolidación de la figura profesional del traductor/intérprete en nuestro país, que apenas empieza a adquirir visibilidad, pasará por una formación de calidad que se vea reflejada posteriormente en la práctica laboral. ${ }^{3}$ Partiendo de estas premisas y

1 Vid. http://www.aneca.es/var/media/150288/libroblanco_traduc_def.pdf

2 Vid. Guía de grado en traducción e interpretación, Universidad de Granada, pág. 2:

"De las tres EUTIs que existian en la década de los ochenta se ha pasado en la actualidad a más de una veintena de centros repartidos por toda la geografía nacional, ya sea dentro de la universidad pública como de la privada: Universidad de las Palmas de Gran Canaria, Universidad Autónoma de Barcelona, Universidad de Málaga, Universidad de Vigo, Universidad Pompeu Fabra, Universidad de Salamanca, Universidad de Vic, Universidad Pontificia de Comillas, CES Felipe II, Universidad Alfonso $X$ El Sabio, Universidad de Alicante, Universidad de Granada, Universidad Jaume I, Universidad de Valladolid, Universidad Europea de Madrid, Universidad del País Vasco, Universidad Autónoma de Madrid, Universidad Antonio de Nebrija, Universidad de Murcia, Universidad Pablo de Olavide, Universidad de Córdoba entre otras."

${ }^{3}$ Tal como indican García Izquierdo, I. y Verdegal, J. (eds.) (1998:9): “...la sociedad civil requiere cada vez con mayor frecuencia productos bien acabados y de calidad 
a fin de proporcionar una base sólida a la formación de futuros profesionales, la investigación en traducción e interpretación adquiere un papel fundamental, especialmente el estudio enfocado a la práctica de la enseñanza de cara a evaluar la pertinencia, eficacia y necesidades de mejora en todos los aspectos del currículum.

El presente trabajo tiene como objeto de estudio las traducciones del alemán al español realizadas por alumnos/as de cuarto curso de la licenciatura en el año académico 2011-2012. En concreto, se estudiará la asignatura Traducción Literaria alemán-español de cuarto, correspondiente a la segunda lengua extranjera, o lengua $C$, dentro del plan de estudios de Traducción e Interpretación de la Universidad de Córdoba. El objetivo es establecer una tipología comparativa de errores traductológicos para los alumnos/as de lengua materna española y alemana que nos permita identificar las necesidades formativas de cada grupo y extraer conclusiones de cara a la evaluación.

\subsection{ENFOQUES ACTUALES EN LA EVALUACIÓN DEL ERROR}

Examinaremos a continuación las propuestas de algunos autores respecto a la clasificación y valoración de errores cometidos por estudiantes de traducción. El enfoque será eminentemente didáctico desde el contexto formativo universitario. No obstante, también interesa relacionar esta formación con la práctica profesional de la traducción, por lo que esta última será un factor a tener en cuenta en la evaluación. No olvidemos que el fin último del proceso de enseñanza es desarrollar en los estudiantes la competencia traductora en todos sus aspectos.

Comenzando precisamente por Hurtado Albir, que como se expone en el punto 2.3 propugna la enseñanza por objetivos de aprendizaje, esta autora distingue entre los siguientes tipos de errores, que ella prefiere denominar "inadecuaciones":

(...) De ahí que la formación de verdaderos expertos en traducción resulte un desafío tan escabroso y apasionante." 
I. INADECUACIONES QUE AFECTAN LA COMPRENSIÓN DEL TEXTO ORIGINAL (contrasentidos, falsos sentidos, sin sentidos, adicion, supresión...)

II. INADECUACIONES QUE AFECTAN LA EXPRESIÓN EN LA LENGUA DE LLEGADA (ortografía y puntuación, gramática, léxico, textual, estilística)

III. INADECUACIONES FUNCIONALES (función textual prioritaria del original, función de la traducción)

Asimismo, su baremo de corrección de traducciones incluye los aciertos, bonificando aquellas equivalencias que se consideran buenas o muy buenas. ${ }^{4}$

Por su parte, Christiane Nord considera la definición de objetivos algo fundamental en la evaluación de traducciones. ${ }^{5}$ Desde esta perspectiva funcional, Nord defiende que el escopo o función de la traducción viene dado por el encargo de traducción:

$\mathrm{Si}$, en el marco del concepto funcional, la finalidad del proceso traslativo se define mediante el encargo de traducción, un nocumplimiento de tal encargo, con respecto a determinados aspectos funcionales, debe considerarse como error o falta. ${ }^{6}$

En cuanto a la jerarquización de los errores, Nord distingue entre errores pragmáticos, errores culturales $y$, en el contexto didáctico, errores lingüísticos. Aunque los errores pragmáticos serían en principio los más graves, dado que "perjudican la funcionalidad directamente al desobedecer las instrucciones pragmáticas del

\footnotetext{
${ }^{4}$ Hurtado Albir, A. (1996:49-50).

5 Vid. Nord, C. (1996:91): “Cualquier definición y evaluación de errores no tiene sentido sin que tomemos como punto de referencia un determinado objetivo educativo claramente definido. En la formación de traductores profesionales, tal objetivo será el siguiente: primero, desarrollar en los estudiantes la capacidad de producir traducciones que 'funcionen', es decir: que correspondan a las necesidades de una comunicación intercultural eficaz, y, segundo, proveerlos de unos criterios racionales para juzgar las traducciones propias y defenderlas, en su caso, frente a los clientes."

${ }^{6}$ Nord, C. (1996:95).
} 
encargo"7, también los errores culturales (p.ej. no respetar las convenciones textuales) y lingüísticos (p.ej. una terminología técnica errónea) pueden afectar negativamente la funcionalidad, adquiriendo así una importancia considerable.

Los errores lingüísticos, que Nord adscribe al contexto didáctico dado que no se puede dar por sentado que los estudiantes dominen ya perfectamente el idioma, merecen una reflexión aparte. ${ }^{8}$ Nord propone que las faltas lingüísticas o estilísticas se marquen de manera diferente al corregir las traducciones de los estudiantes. Asimismo, esta autora señala que la puntuación de los errores de traducción depende del encargo. Por ejemplo:

en una traducción acabada, una falta tipografica puede constituir un error gravísimo, mientras que en una traducción-resumen la misma falta no cuenta casi nada. ${ }^{9}$

Otros autores, como Kussmaul y Hönig, adoptan un enfoque cognitivista a la hora de evaluar las traducciones de sus estudiantes. Así, Kussmaul propone el "punto de vista del traductor profesional" en la evaluación de traducciones, y no exclusivamente el del "profesor de idiomas"10. Basándose en los conceptos de Pym de "error binario" (los que indudablemente son errores) y "error no binario" (que se basa en la selección entre varias opciones de traducción), Kussmaul introduce junto con Hönig the maxim of the sufficient degree of precission, un enfoque cuantitativo del error que cuestiona el alcance del mismo o, en otras palabras, el grado de precisión que debe tener la traducción

\footnotetext{
${ }^{7}$ Nord, C. (1996:99).

${ }^{8}$ Nord, C. (2006:37): "(...) en las traducciones de los alumnos no solo encontramos errores de traducción sino también insuficiencias en la lengua meta (LM) que no se pueden explicar por la influencia de la lengua del original. Para ayudar a los alumnos a mejorar su competencia lingüística, proponemos marcar estas faltas, pero hay que hacer hincapié en que no se confundan con errores de traducción." ${ }^{9}$ Nord, C. (2006:39).

10 Vid. Kussmaul, P. (1995:128): "Error assessment is focused on the communicative function of the word, phrase or sentence in question. Distortion of meaning must be seen within the text as a whole and with regard to the translation assignment and the receptor of the translation. (...) It may very well be that what is a mistake from view."
} 
ofrecida para cumplir su propósito. Kussmaul clasifica los errores en problemas culturales, situacionales, de actos de habla, de significado y problemas lingüísticos. Sin embargo, esta clasificación es más una ayuda para los estudiantes que una jerarquización del error con penalizaciones establecidas para cada categoría:

They will be able to see which areas they have to concentrate on. ${ }^{11}$

Para calificar todos los tipos de error, debería recurrirse a la máxima del suficiente grado de precisión. Al igual que Hurtado Albir, Kussmaul es partidario de evaluar positivamente aquellos pasajes de una traducción que hayan sido resueltos satisfactoriamente.

Por su parte, Hönig también aboga por una evaluación de las traducciones basándose no únicamente en encontrar el equivalente exacto de una palabra (ya que una traducción encontrada en un diccionario puede ser errónea según el contexto), sino en que el texto traducido "funcione" para el receptor en un contexto real o profesional. ${ }^{12}$ Por tanto, la precisión en la terminología, así como la literalidad de una traducción, deberán ser evaluadas según el tipo de texto y el objetivo del mismo:

Es kann nur einen Maßstab für übersetzerisches Handeln geben: Einen Text abzuliefern, der für die (zuvor präzise definierte) Gruppe von Adressaten funktionsfähig ist.13

Como vemos, todos estos autores tienen en común la evaluación de traducciones en un contexto dado y más allá de los meros problemas de equivalencia o de corrección lingüística.

${ }^{11}$ Kussmaul, P. (1995:153).

12 A este respecto, indica Hönig, H.G. (1995:130): "Man kann nicht einerseits behaupten, man bilde zum professionellen Übersetzer aus, und andrerseits bei der Bewertung von Übersetzungsfehlern Kriterien verwenden, die eigentlich auf ein Abfragen von Vokabeln hinauslaufen".

${ }^{13}$ Hönig, H.G. (1995:137).

Futhark 9 (2014)

Castillo, Análisis de las causas de errores, 35-64

ISSN 1886-9300 


\subsection{EL HORIZONTE DE EXPECTATIVAS}

Un último concepto que resulta interesante en la presente investigación es el denominado "horizonte de expectativas":

A base de encargos de traducción, que durante el periodo de aprendizaje serán supuestos didácticos encaminados a comprobar si los alumnos han logrado los objetivos de aprendizaje previstos para la fase en cuestion, el docente formula un horizonte de expectativas, especificando el rendimiento que se espera de los alumnos. Dicho horizonte de expectativas toma en consideración el grado de dificultad de la tarea y define lo que será marcado como 'error' en la evaluación del trabajo. ${ }^{14}$

Nord indica que una tarea de traducción tiene que ser factible, por lo que las dificultades que plantee no deben ir más allá de los conocimientos y competencias del alumnado en el momento de realizarla. En nuestro caso, esto se traduce en que las pruebas de traducción que realicen nuestros alumnos/as deben basarse en las traducciones y los conceptos teóricos que ya se han visto en clase. Durante el diseño de la prueba, se tienen en cuenta estos puntos.

De forma paralela a su clasificación de los errores de traducción, Nord distingue entre dificultades de traducción pragmáticas, culturales y lingüísticas. Una cuarta categoría serían los problemas de traducción concretos presentados por un texto específico, que no forman parte de la sistematización de las dificultades por no ser generalizables. Dado que los problemas de traducción afectan no solo a los estudiantes sino a los traductores profesionales, dichos problemas deberían ser limitados en las tareas o pruebas propuestas que realicen los estudiantes, hasta fases más avanzadas de su formación.

\section{METODOLOGÍA}

La didáctica de la traducción pertenece al ámbito de la investigación aplicada a la traducción, siendo quizá el campo más

14 Nord, C. (2006:35) 
característico de la misma, como destaca Hurtado Albir. ${ }^{15}$ La autora distingue asimismo diferentes tendencias en este campo de estudio, con especial mención a las propuestas de la enseñanza por objetivos de aprendizaje introducidas por J. Delisle. ${ }^{16} \mathrm{El}$ presente trabajo se enmarca en este último punto, por lo que el análisis de los errores de traducción que se llevará a cabo y las propuestas evaluativas que se realizarán estarán relacionados en todo momento con los objetivos planteados en el currículum y en las líneas de investigación escogidas.

\subsection{CONTEXTUALIZACIÓN}

Traducción Literaria alemán-español es una asignatura obligatoria y cuatrimestral, de 6 créditos, impartida en el $4^{\circ}$ curso de la licenciatura. Los contenidos se agrupan en los siguientes bloques:

1. Terminología y conceptos básicos

2. El drama en la literatura en alemán

3. La narrativa en la literatura en alemán

4. La novela corta, el cuento y el relato en la literatura en alemán

5. La lírica en la literatura en alemán

6. La literatura en alemán y el cine: adaptaciones cinematográficas

El objetivo fundamental de la asignatura es:

Posibilitar al alumno unos conocimientos básicos de Literatura Alemana para poder acceder a la traducción de textos literarios alemanes al español. ${ }^{17}$

\footnotetext{
15 Hurtado Albir, A. (2001): Traducción y traductología. Introducción a la Traductología, citado por Ruiz Yepes (2006:15).

16 Hurtado Albir, A. (1996: 33).

17 Guía docente de Traducción Literaria alemán-español, Licenciatura en Traducción e Interpretación, Universidad de Córdoba.
} 
En efecto, podemos asumir que alumnos/as estudiantes cuentan ya con conocimientos traductológicos adquiridos en asignaturas que han cursado con anterioridad:

(...) se puede esperar de este estudiante cierta madurez intelectual que ha tenido que ir adquiriendo a lo largo de la carrera y que le permite saber, por ejemplo, cómo organizar su trabajo. ${ }^{18}$

La consecuencia práctica es que se incidirá en menor medida en la adquisición de destrezas traductológicas generales en el caso de esta asignatura, concentrándonos en las particularidades de la traducción de textos literarios en lengua alemana. La finalidad es que los estudiantes conozcan los principales autores y géneros y cómo abordar la traducción de los mismos. Más allá de la práctica profesional, resulta imprescindible completar la formación en una lengua extranjera con nociones sobre la literatura en dicha lengua y la herencia cultural que supone.

El planteamiento del trabajo en esta asignatura prevé que se inicie cada bloque temático con una introducción teórica sobre la época literaria y los géneros que se van a tratar. A continuación, se examinan varias obras en clase, sus traducciones e información relativa a las mismas. Los estudiantes trabajan en ocasiones de manera autónoma y otras veces por grupos, a veces grupos mixtos con distinta lengua materna. Es una clase de tan solo 11 estudiantes, por lo que permite un trato personalizado y un trabajo colaborativo.

Las pruebas de cada bloque en el marco de la evaluación continua consisten en una traducción de una obra vista en clase. Asimismo, la traducción de la prueba es, siempre que sea posible, un extracto inmediatamente anterior o posterior al fragmento de la obra traducido $y$ visto en clase. De esta manera, los alumnos deberían estar perfectamente familiarizados con el tema que se les presenta, las características de la obra y lo que se espera de ellos. La duración de la prueba es de $2 \mathrm{~h}$., está permitido el uso del material de clase, diccionarios y otras obras, pero no hay acceso a internet. A los alumnos se les presenta el capítulo o acto entero en cuestión, con el fragmento que tienen que traducir en negrita. De esta manera, los

${ }^{18}$ Martínez, L. (2007: 400). 
estudiantes cuentan con el contexto completo, en caso de que no recuerden el fragmento.

El encargo de traducción que los alumnos reciben es la traducción de los textos conforme a las convenciones literarias de cada tipología textual, con el fin de aparecer en una publicación sobre literatura en lengua alemana en sus diversas épocas y géneros. Dado que tanto las características del texto origen como las que se esperan del texto meta han sido vistas en clase con las mismas obras, se presupone que los estudiantes están familiarizados con ellas.

Los dos bloques temáticos que examinados en el presente trabajo son el bloque II, el drama, y el bloque III: la narrativa. Se analizaron los errores cometidos por los alumnos de ambos idiomas en las pruebas correspondientes a cada bloque.

En la prueba 3, presentamos a los alumnos un extracto del drama burgués Emilia Galotti, de Gotthold Ephraim Lessing. ${ }^{19}$ Representado por primera vez en 1772, pertenece a la época de la Ilustración alemana. Se trata de una obra novedosa que rompe con las características del teatro francés, modelo de su tiempo, para presentar esencialmente una reivindicación política contra la nobleza y a favor de la burguesía, adalid de los nuevos ideales y del nuevo concepto de la moral.

En cuanto a la prueba 4, es un extracto de El Proceso de Franz Kafka. ${ }^{20}$ Se trata de una obra inacabada, publicada póstumamente en 1925. Es una novela compleja y difícil de clasificar, aunque se le han

19 Disponible online en el $\begin{gathered}\text { Proyecto } \\ \text { Gutenberg: }\end{gathered}$
http://gutenberg.spiegel.de/buch/1174/1. En el drama Emilia Galotti., el
fragmento de la prueba pertenece a la primera escena del acto tercero, en el que el
príncipe de Guastalla conversa con su chambelán, Marinelli. Hablan de Emilia
Galotti, prometida del conde Appiani, de la que el principe se ha encaprichado. El
príncipe reprocha a Marinelli su incapacidad para ayudarle a ganarse los favores
de Emilia, momento en el que se oyen unos disparos. Este principio de la escena
había sido traducido en clase, y los alumnos deben traducir en la prueba la
siguiente secuencia, en la que Marinelli explica al príncipe su plan para raptar a
Emilia, que se llevando a cabo en ese momento.
20 Disponible ondine en el Proyecto
http://gutenberg.spiegel.de/buch/157/1. Gutenberg: 
atribuido tintes políticos anarquistas o filosófico-existencialistas, entre otros.

\subsection{CLASIFICACIÓN DEL ERROR}

En el caso de la traducción literaria, la corrección del texto meta es más compleja que en traducción general. Se trata de que la versión española cumpla con las expectativas del lector o espectador hispanohablante. En este sentido, el lenguaje y el estilo deben ser adecuados a la época y al tipo de obra. Asimismo, se debe transmitir el sentido del fragmento, sin perder de vista que la función predominante del mismo no es la referencial e instrumental, sino la estética. Es decir, no estamos buscando la precisión en la traducción de la terminología de cara a la aplicación práctica de su contenido. Más bien buscamos el entretenimiento del lector, su comprensión del contexto y de la época y, en última instancia, de las ideas que desea transmitir el autor. Esto requiere de los alumnos un gran esfuerzo, puesto que no es la línea predominante en los estudios de traducción y les exige una extensa preparación previa para familiarizarse con el autor, la época literaria y las características de la obra.

Siguiendo la propuesta de Hurtado Albir, las inadecuaciones detectadas se clasificaron en errores de sentido, errores lingüísticos y errores pragmático-funcionales. Los errores de sentido afectan a la comprensión del original y su traspaso a la lengua meta, se trata de contrasentidos, falsos sentidos, sin sentidos y léxico incorrectamente traducido. Las adiciones y omisiones también pertenecen a esta categoría general, si bien, dependiendo de la repercusión del error, podrían considerarse también como errores pragmático-funcionales. Los errores lingüísticos afectan a la lengua meta: errores en el régimen de preposiciones, etc., errores de concordancia de género o número, ortografía, puntuación y gramática. Los errores pragmáticofuncionales, que afectan a las convenciones de género y tipología textual, así como a la función de la traducción, son los relacionados con la ortotipografía, redacción, estilo y presentación. 


\subsection{METODOLOGÍA DE ANÁLISIS}

Se analizaron un total de 45 fragmentos o unidades de sentido "problemáticas" en las dos pruebas y se extrajeron las traducciones de seis alumnos, tres de lengua materna española y tres de lengua materna alemana. Para identificarlos de manera anónima de manera previa al análisis, se asignó una letra de la $\mathrm{A}$ a la $\mathrm{F}$ a cada estudiante, a continuación se indica su lengua materna (ES o DE) y el número de la prueba que realizo (pruebas 3 y 4 de Traducción Literaria), de la siguiente manera: ADE3, BDE3, DES4, etc. Una vez identificadas las respectivas traducciones, se procede a clasificarlas en las distintas categorías y a examinar las posibles causas de cada error.

Cabe señalar que esta investigación se enmarca en un análisis más extenso con dos pruebas adicionales (prueba 1 y prueba 2) correspondientes a la asignatura de Traducción General alemánespañol. En el presente trabajo se exponen únicamente los resultados correspondientes a los errores de sentido detectados en las pruebas de la asignatura de Traducción Literaria.

\section{RESULTADOS}

El propósito este trabajo es establecer una comparación entre la tipología de error en traducciones de alumnos germanoparlantes e hispanohablantes. A tal fin, se indicará en primer lugar una comparación cuantitativa de los errores constatados. No se trata de realizar una mera cuantificación de los errores, ya que solo un análisis cualitativo en contexto ofrecerá datos relevantes para la mejora del proceso de enseñanza-aprendizaje y para la evaluación. No obstante, se pueden extraer algunas conclusiones interesantes de la incidencia de cada tipo de error en los dos grupos de alumnos.

De los 51 errores de sentido detectados, los alumnos germanoparlantes realizaron un total de 12 en la prueba 3 y 3 errores en la prueba 4 , mientras que los hispanohablantes tuvieron un total de 20 errores en la 3 y 16 en la prueba 4 . A priori, se aprecia una disminución de la concentración de errores en ambos grupos a 
medida que progresa la asignatura. La subcategoría que más errores registró es la correspondiente a la desviación del sentido o falsos sentidos, en la que los alumnos germanoparlantes registraron 4 errores en la prueba 3 y 0 en la prueba 4 , mientras que los hispanohablantes obtuvieron 15 errores en la prueba 3 y 9 en la 4 .

A continuación, se examinará de forma detallada cada categoría. A los principales ejemplos añadiremos en cada caso las observaciones realizadas en clase y la retroalimentación proporcionada por los mismos estudiantes:

\subsection{CONTRASENTIDOS}

Dado que es uno de los errores más graves que pueden encontrarse en una traducción, tanto por sus repercusiones como porque indica una pobre competencia por parte del traductor, es alentador observar que únicamente se dio un caso en las pruebas estudiadas. Es el caso de un alumno germanoparlante en la prueba 3:

T.O.: Und ein anderer Teil, wobei einer von meinen Bedienten ist, wird aus dem Tiergarten gestürzt sein ...21

CDE3: Y la otra parte, lo que uno es de mis ayudantes, será lanzado al parque...

Aquí, el sentido del verbo es 'precipitarse, llegar repentinamente'. El alumno germanoparlante ha escogido la acepción incorrecta en el diccionario (ya que stürzen también significa 'arrojar') y asimismo ha tenido dificultades con la elección del tiempo verbal, empleando el futuro de la voz pasiva en lugar del futuro perfecto de la voz activa.

${ }^{21}$ En este fragmento, el chambelán del Príncipe de Guastalla, Marinelli, explica al Príncipe cómo ha dividido a sus hombres en dos grupos: el primero habrá fingido atacar el carruaje donde viaja Emilia y el segundo grupo, donde se encuentra uno de los hombres de confianza de Marinelli, se habrá precipitado desde el parque del Príncipe como si acudiera al rescate (Emilia Galotti, tercer acto, escena primera). 


\subsection{FALSOS SENTIDOS}

Este es uno de los puntos de mayor importancia, especialmente para los alumnos hispanohablantes. Observamos una mayor concentracion de errores la prueba 3 . Se trata de la primera traduccion de la asignatura realizada por los estudiantes en condiciones de examen (ya que la primera prueba de esta asignatura no consistía en una traducción sino un examen de conceptos introductorios). Esto posiblemente explique una mayor incidencia de errores respecto a la prueba 4.

El texto original presenta la dificultad inicial del enlace con el texto anterior (traducido en clase). En efecto, las primeras frases son alusiones a lo que acontece inmediatamente antes (el sonido de disparos), y por tanto solo es posible traducirlas correctamente teniendo en cuenta ese contexto. La primera frase, en la que el príncipe pregunta qué sucede, no plantea problemas a los alumnos. En la segunda, por el contrario, encontramos las siguientes traducciones:

T.O.: Marinelli. Was meinen Sie wohl?22

DES3: ¿Se encuentra bien?

EES3: ¿Estáis bien?

FES3: ¿Se encuentra bien, su excelencia?

Aquí se introducen falsos sentidos por la incorrecta traducción del verbo meinen (pensar, opinar) y de la partícula wohl (posible, probable), en este último caso por confusión con el adjetivo homógrafo (wohl=bueno, bien).

Los falsos sentidos a menudo se producen por la traducción literal o errónea de una palabra:

22 El Príncipe, asustado por los disparos, pregunta qué está sucediendo, a lo que Marinelli responde irónico: “¿Qué pensáis vos que puede estar sucediendo?" [traducción libre]. Los alumnos no tuvieron dificultades al traducir la pregunta del Príncipe, pero no consiguen interpretar correctamente la respuesta irónica de Marinelli. 
Marinelli. Kurz: wovon ich gesprochen, geschieht.

EES3: Corto: Hablo de lo que ha sucedido.

Aquí, el sentido de kurz es 'en pocas palabras'.

Encontramos numerosos ejemplos de errores de sentido provocados por el uso incorrecto o insuficiente del diccionario. Un caso en el que la mayoría de los alumnos tuvo dificultades es el siguiente:

Der Weg geht hart an der Planke des Tiergartens vorbei.

DES3: El camino se torna difícil cerca de la empalizada del parque.

FES3: El duro sendero que recorre la empalizada pasa por el parque.

CDE3: El camino pasa duro por la empalizada del parque.

En este caso, hart no corresponde al adjetivo 'duro, difícil', sino que junto a la preposición an constituye un adverbio con el significado 'cerca, rayando'. Probablemente, esta acepción no estaba incluida en los diccionarios empleados por los alumnos, debido a su escasa frecuencia de uso en alemán. También es posible que los alumnos simplemente tomaran la primera acepción, sin detenerse a cuestionar su adecuación en la frase.

Examinaremos a continuación los errores de sentido en las traducciones de la prueba $4 . .^{23}$ Curiosamente, encontramos de nuevo falsos sentidos relacionados con el verbo meinen:

T.O.: „Es war gut gemeint" 24

\footnotetext{
${ }^{23}$ El protagonista, Joseph $\mathrm{K}$., se encuentra en su domicilio, una habitacion alquilada a su casera, la señora Grubach, cuando es detenido por dos desconocidos, sin que medie explicación ni él sepa el motivo de esta detención. Los alumnos deben traducir el fragmento en el que, mientras uno de los desconocidos permanece en su habitación, el protagonista se dirige a la habitación contigua para hablar con el segundo (El proceso, capítulo 1).
} 
DES4: Bien pensado.

FES4: Era un canalla de los buenos.

En ninguna de estas dos traducciones se interpreto correctamente que el personaje quería declarar sus buenas intenciones. El primer estudiante toma una acepción del verbo que no es la adecuada en este caso, mientras que el segundo confunde gemeint (participio de meinen, tener intencion) con gemein (malvado).

La descripción de la habitación contigua plantea numerosos problemas a los estudiantes, dado que las frases son largas y presentan un conector complejo:

T.O.:...vielleicht war in diesem mit Möbeln, Decken, Porzellan und Photographien überfüllten Zimmer heute ein wenig mehr Raum als sonst, man erkannte das nicht gleich, um so weniger, als die Hauptoeränderung in der Anwesenheit eines Mannes bestand...

La idea es que 'la habitación, sobrecargada de muebles, quizá hoy parece más espaciosa que de costumbre, pero no se identificaba este hecho inmediatamente, tanto menos cuando el cambio principal residía en la presencia de un hombre' [traducción libre].

El conector um so weniger es interpretado por los alumnos como sigue:

DES4: ...la habitación estaba irreconocible; al menos, el cambio principal residía en la presencia de un hombre...

EES4: ...estaba abarrotado de muebles, mantas, piezas de porcelana y fotografías aunque aquel día estaba más espacioso y uno no lo podía reconocer, el principal cambio sería un hombre...

En el primer caso, el estudiante emplea un conector que es un sin sentido en esta frase. El segundo estudiante omite el conector, alterando ligeramente el estilo pero sin introducir una desviación. En

${ }^{24} \mathrm{El}$ primer desconocido pregunta a $\mathrm{K}$. si no prefiere permanecer en el cuarto, y ante la respuesta negativa de $\mathrm{K}$. le indica que lo había sugerido con buena intención (El proceso, capítulo 1 ). 
cuanto a los alumnos germanoparlantes, tuvieron dificultades al formular correctamente la traducción del conector:

CDE4: ...no era posible observarlo en seguida, tanto menos el cambio central era la presencia de un hombre...

ADE4: ...esto no se notó enseguida, tanto menos como el cambio principal ahora consistía en la presencia de un hombre...

También la frase introductoria de este párrafo planteo algunas dificultades:

T.O.: Im Nebenzimmer, in das K. langsamer eintrat als er wollte, sah es auf den ersten Blick fast genau so aus, wie am Abend vorher.

FES4: En el cuarto vecino en su lentitud, K. a primera vista vio casi exacto el registro que quería...

El estudiante no identifica ambos comparativos y por tanto no reproduce el sentido de que $\mathrm{K}$. entró en la habitación con más lentitud de lo que habría deseado, así como que todo parecía casi igual que la tarde anterior. En otro ejemplo del mismo fragmento:

DES4: $K$ entró lentamente en la habitación contigua y dio un vistazo rápido inicial tal y como había hecho la tarde anterior.

Se trata, en general, de errores de menor alcance que en la prueba 3. En su mayor parte, los estudiantes reproducen con éxito la escena descrita por el autor.

\subsection{SIN SENTIDOS}

Se detectaron algunos casos, uno en la prueba 3. Aquí estudiante intenta reproducir las palabras sin conseguir recrear la escena del original, ya que no ha entendido las estructuras de la lengua origen ni
la descripción:

T.O.: Und ein anderer Teil, wobei einer von meinen Bedienten ist, wird aus dem Tiergarten gestürzt sein; den Angefallenen gleichsam zur Hilfe. 
EES3: Y la segunda, en la cual hay que estar muy atentos, es cuando esté perturbada en el parque, en cierto modo se le atacará en la ayuda.

En este caso, el estudiante tuvo dificultades con Bedienten (sirvientes), wird gestürzt sein (se habrá precipitado, habrá llegado repentinamente) y Angefallenen (los asaltados). El sentido está completamente distorsionado y parece probable que el estudiante tenga dificultades para reconocer las estructuras verbales y la sustantivacion de participios.

Para ilustrar la causa de estos problemas, recurriremos a la teoría de scenes and frames de Fillmore (1977), según la aplicación realizada por Snell-Hornby al campo de la traducción. Tal como explica esta autora, según Fillmore los signos linguísticos que codifican un texto son frames, mientras que scene sería la situación recreada por dichos signos. ${ }^{25}$

En el caso de los alumnos hispanohablantes, los frames del texto de la prueba 3 plantean numerosas dificultades, en primer lugar por el vocabulario. Palabras como Bedienter y Angefallene, que se derivan de verbos $\mathrm{y}$ por tanto no se encuentran como tal en el diccionario, $o$ acepciones distintas de la habitual como en la palabra hart, son algunos de los escollos a los que estos alumnos se enfrentaron. La causa es, por un lado, un escaso dominio de las normas gramaticales correspondientes del alemán, y por otro la incapacidad de imaginar la escena descrita más allá de las palabras. En efecto, una traducción de la frase omitiendo la palabra hart hubiera sido más adecuada que escoger una acepción claramente errónea.

De nuevo, es de destacar que los alumnos germanoparlantes también tuvieron dificultades con esta palabra, traduciéndola por su

25 Vid. Snell-Hornby, M. (1995: 81): "The translator starts from a presented frame (the text and its linguistic components); this was produced by an author who drew from his own repertoire of partly prototypical scenes. Based on the frame of the text, the translator-reader builds up his own scenes depending on his own level of experience and his internalized knowledge of the material concerned. As a nonnative speaker, the translator might well activate scenes that diverge from the author's intentions or deviate from those activated by a native speaker of the source language (a frequent cause of translation error)." 
primera acepción. No obstante, mientras que los hispanohablantes fallan al activar la escena mental correspondiente, el problema para los alumnos de habla materna alemana reside en expresar correctamente dicha escena en la lengua meta:

Based on the scenes he has activated, the translator must now find suitable TL-frames; this involves a constant process of decisionmaking, whereby he depends entirely on his proficiency in the target language. ${ }^{26}$

Como vimos en el ejemplo del contrasentido, los errores de expresión en la lengua meta a menudo desembocan en falsos sentidos: aunque se intuya que el alumno ha reproducido la escena mental correctamente para sí, el resultado final no lo demuestra, y un lector del texto meta tampoco recibiría la misma escena que el lector del original.

En cualquier caso, también los falsos sentidos en la traducción de las primeras frases de la prueba 3 (el intercambio entre Marinelli y el príncipe) nos indican que los estudiantes encuentran el contexto difícil de comprender: las relaciones entre ambos personajes, las intenciones de cada uno de ellos, el sentido final que intenta transmitir el autor, siempre relacionado con la crítica de la aristocracia. A este respecto, cabe indicar que, durante las clases de traducción literaria, uno de los estudiantes germanoparlantes reiteraba frecuentemente su escasa comprensión del original. Este estudiante, por ser de nacionalidad austriaca, probablemente tuviera una experiencia muy limitada con obras de la literatura alemana.

\subsection{LÉXICO}

En el caso de esta asignatura podríamos matizar que los errores no son usos inadecuados de los términos especializados (terminología), sino más bien inadecuaciones en cuanto a la acepción o el significado más preciso de una palabra.

${ }^{26}$ Snell-Hornby, M. (1995: 81).

Futhark 9 (2014)

ISSN $1886-9300$

Castillo, Análisis de las causas de errores, 35-64 
Los estudiantes germanoparlantes tienen algunos problemas al escoger la traducción más adecuada para un término concreto:

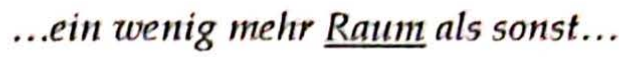

CDE4: ...tendríamos hoy más lugar que normalmente...

Aquí, el estudiante escogio 'lugar' en vez de 'más espacio que de costumbre'. En otro ejemplo:

T.O.: ...als die Hauptveränderung in der Anwesenheit eines Mannes bestand...

BDE4: ...el cambio principal que era la asistencia de un hombre...

En lugar de 'presencia', el estudiante indica 'asistencia', de nuevo una confusión con las acepciones encontradas en el diccionario.

También encontramos algún ejemplo de términos mal seleccionados en el caso de los alumnos hispanohablantes:

T.O.: Es war das Wohnzimmer der Frau Grubach...

FES4: Era el cuarto donde se encontraba la señora Grubach...

EES4: Era la habitación de la señora Grubach...

Aquí es necesario que Wohnzimmer se traduzca como 'cuarto de estar' o 'salón', puesto que de lo contrario no se transmite el significado correcto en el contexto de que el protagonista había alquilado una habitación en casa de la señora Grubach, a cuyo salón él tiene acceso. Los tres alumnos germanoparlantes tradujeron correctamente 'salón'.

Como ya hemos matizado en alguna ocasión, cada tipo de texto tiene sus propios requisitos de cara a su función y a la satisfacción del lector. Siguiendo la clasificación de Reiss (1971), podemos distinguir entre:

1. "inhaltsbetonte Texte" en los que predomina la función de representación de la lengua, y que transmiten ante todo un contenido; 
2. "formbetonte Texte", textos en los que domina la funcion expresiva de la lengua: la forma de expresión es tan importante o más que el contenido.

3. "appellbetonte Texte" son aquellos cuya funcion principal estriba en la "llamada" al lector o receptor, en producir en éste la reacción deseada por el autor. ${ }^{27}$

La traducción literaria, se adscribe a la categoría 2 de Reiss (textos expresivos en los que la forma es igual o más importante que el contenido). En este sentido, aunque dependa en menor medida de la exactitud terminológica, ocurre que la elección errónea de una palabra (como en el caso de 'habitación' por Wohnzimmer en la prueba 4 puede afectar al sentido general del texto y a la comprensión. El problema de los alumnos germanoparlantes en esta asignatura es también seleccionar la traducción adecuada entre las ofrecidas en el diccionario (recuérdese el ejemplo de 'asistencia' en lugar de 'presencia', también en el texto 4).

\subsection{ADICIONES}

Afectan únicamente a los alumnos hispanohablantes en el estudio que nos ocupa. Se trata de expresiones de cosecha propia, redundantes o que afectan a la función del texto al añadir información innecesaria o confusa para el lector:

Im Nebenzimmer, in das K. langsamer eintrat als er wollte, sah es auf den ersten Blick fast genau so aus, wie am Abend vorher.

FES4: En el cuarto vecino, en su lentitud, $\mathrm{K}$. a primera vista vio casi exacto el registro que quería, así con los planes para aquella noche.

El estudiante introduce una adición de cosecha propia ('los planes'). En otro ejemplo del mismo fragmento:

DES4: $K$. entró lentamente en la habitación contigua y dio un vistazo rápido inicial tal y como había hecho la tarde anterior.

${ }^{2}$ Elena García, P. (1990: 38-39) 
Aquí, el estudiante omite también que K. entró más lentamente de lo que pretendía, e introduce el falso sentido de que echó un vistazo tal y como la tarde anterior, en lugar de que todo parecía casi igual que la tarde anterior. Con toda probabilidad, la causa es que el alumno no ha reconocido el verbo separable aussehen (parecer, tener aspecto) y por tanto ha traducido el verbo sehen (ver).

\subsection{OMISIONES}

Se detectaron dos:

T.O.: Im Nebenzimmer, in das K. langsamer eintrat als er wollte, sah es auf den ersten Blick fast genau so aus, wie am Abend vorher.

FES4: En el cuarto vecino, en su lentitud, $K$. a primera vista vio casi exacto el registro que quería, así con los planes para aquella noche.

El estudiante omite el sentido de que $\mathrm{K}$. entró en la habitación con mayor lentitud de lo que habría deseado, al no reconocer la estructura comparativa langsamer als.

En cuanto al caso del alumno germanoparlante, se trata de la omisión de un adverbio:

T.O.: Da wird ein Teil den Wagen angefallen haben; gleichsam, um ihn zu plündern.

ADE3: Una parte habría atacado el coche para despojarlo...

Aquí, en lugar de indicar 'en apariencia, fingiendo', gleichsam se omite, causando una pérdida de significado. Al preguntar al alumno por la omisión, indicó que no le fue posible encontrar gleichsam en el diccionario, por lo que decidió omitirlo.

El motivo de una omisión no estratégica puede indicar el desconocimiento del significado de una expresión o bien un descuido por parte del estudiante. En cualquier caso, es un fallo relativamente 
facil de detectar por un revisor o cliente, por lo que los estudiantes deberian tener especial cuidado en evitarlo. En este caso, la solución seria emplear un diccionario bilingüe más completo.

\section{CONCLUSIONES}

Los objetivos del presente trabajo comprenden el análisis de las causas de los errores, la puntuación de los mismos y las directrices para el trabajo de clase. No es nuestra intención, en este punto, formular conclusiones universales, ni mucho menos prescriptivas. Los resultados del presente estudio se enmarcan en un contexto concreto y su propósito es contribuir al proceso de enseñanza-aprendizaje que tiene lugar en la asignatura descrita. El estudio de las pruebas seleccionadas es limitado y no puede ser representativo de los estudiantes de otras materias o planes docentes. Sí esperamos, por el contrario, que las conclusiones que a continuación formularemos sean de utilidad para la impartición de esta asignatura en el futuro.

\subsection{CAUSA DE LOS ERRORES DETECTADOS}

Entre las causas de error más recurrentes en los alumnos hispanohablantes se encuentra el análisis morfologico y morfosintáctico deficiente del T.O. Afecta especialmente a las estructuras verbales, a los conectores y a la morfología de los sustantivos cuando estos se derivan de participios, entre otros. En combinación con este punto, el uso inadecuado del diccionario o la inadecuación del diccionario en sí (extensión reducida, falta de uso de diccionarios monolingües) resulta un problema añadido que afecta tanto a los alumnos hispanohablantes como a los germanoparlantes. Ambos grupos presentan asimismo confusión entre palabras homógrafas o de grafía similar.

En el caso de los alumnos germanoparlantes, la expresión errónea en la lengua meta produce falsos sentidos e incluso contrasentidos. En 
concreto los tiempos verbales ${ }^{28}$ y ciertas estructuras como los conectores presentan dificultades a los estudiantes no nativos.

Las carencias de ambos grupos se ven acentuadas por la falta de preparación de las obras y el autor, así como de contextualización de las escenas que deben traducir.

\subsection{TIPOLOGÍA Y PUNTUACIÓN DE LOS ERRORES}

La corrección de las pruebas basándonos en una clasificación de los errores contribuye al objetivo de afianzar los conocimientos de lengua de los estudiantes, así como la competencia traductora. En efecto, es necesario indicar a los estudiantes por qué un error se considera como tal y cuál ha sido el fallo en el proceso traductor que ha llevado a cometerlo. Sin embargo, esto no significa que cada categoría tenga una puntuación fija de cara a obtener la nota de una traducción. Proponemos, por el contrario, una puntuación variable del error dependiendo en las repercusiones del mismo, siguiendo el enfoque funcionalista de Nord, así como el punto de vista del traductor profesional (Kussmaul) y la máxima del suficiente grado de precisión (Kussmaul y Hönig). Asimismo, abogamos por la bonificación en el caso de resolución acertada de problemas de traducción (Hurtado Albir, Kussmaul).

El sistema de evaluación del error empleado en Traducción Literaria se basa el baremo empleado por Balbuena Torezano y Cobos en los manuales de ambas asignaturas. ${ }^{29}$ En el presente trabajo se

${ }^{28}$ Balbuena Torezano, M.C. (2006:181): "Más complicado resulta, para el traductor germanoparlante, el dominio de los tiempos verbales. Así, es frecuente que en un mismo párrafo se empleen de forma incorrecta distintos tiempos de pasado junto a otros de presente, o bien que se traduzcan de forma incorrecta los tiempos pasados. El traductor ha de tener en cuenta, dentro del pretérito, que, en español, se ha de emplear el pretérito perfecto para indicar aquellas acciones pasadas que sin embargo están muy cercanas al presente, y que el pretérito imperfecto, por su parte, sirve para indicar acciones que se realizaban en el pasado de forma repetida o con cierta frecuencia."

29 Balbuena Torezano, M.C. (2011:127); Balbuena Torezano y Cobos López, I. (2011:92): las categorías del error son los errores de transmisión de sentido 
propone una clasificación ligeramente diferente, según las categorías expuestas en el punto 2.2. La penalización de cada error sería como sigue: de 0,25 a 1 punto para los errores de sentido y los lingúísticos, $y$ de 0,5 a 2 puntos por los errores pragmáticos y funcionales, por entender que estos últimos son los de mayor repercusión en el grado de adecuación de la traducción correspondiente a la función que debe cumplir.

En esta clasificación, la puntuación equivalente de los errores de sentido y los errores lingǘsticos garantiza que los estudiantes hispanohablantes (que tienden a acumular inadecuaciones de sentido) y los germanoparlantes (que generalmente presentan más errores lingüísticos) puedan obtener resultados similares sin verse perjudicados por un sistema de evaluación que dé más importancia al contenido o a la forma. Del mismo modo, los errores pragmáticos y funcionales serán los más penalizados, puesto que consideramos que con una preparación suficiente de los contenidos de clase ambos grupos pueden obtener resultados satisfactorios.

La clasificación de los errores es igualmente flexible. A modo de ejemplo, un error léxico o gramatical especialmente grave puede constituir error pragmático o funcional, en cuyo caso su penalización sería mayor.

Del mismo modo, dependiendo del tipo de texto daremos mayor importancia a unos aspectos u otros. En el caso de un texto técnico, la precisión y corrección en la terminología será primordial, mientras que en un texto literario narrativo el estilo y la redacción primarán sobre una traducción literal de una expresión. No obstante, estos no son criterios absolutos, puesto que hemos visto ejemplos en los que un error léxico podía perjudicar la comprensión de un texto literario.

(penalizados con un máximo de 2 puntos) y los de expresión en la lengua de llegada (penalización de hasta 2 puntos para errores léxicos y morfosintácticos $\mathrm{y}$ de hasta 1 punto para errores formales, ortográficos y de redacción). 


\subsection{DIRECTRICES PARA EL TRABAJO EN CLASE}

El principal escollo constatado en la gestión del trabajo de clase es la utilización del material de referencia. Aunque se incluyen en el dossier que forma parte de la evaluación de cada bloque temático, su misión fundamental es servir de apoyo durante las pruebas, ya que incluyen la contextualización de los textos. No obstante, como se ha descrito en apartados anteriores, hemos observado que los estudiantes no extraen el provecho deseado de estos materiales. En Traducción Literaria, los estudiantes deben aprender a estructurar la información de cada obra vista en clase en su material de referencia. De este modo, cuando se les presente el texto de prueba podrán consultar más fácilmente el contexto de dicho fragmento. Se les propondrá que, además del breve resumen de la obra y de la vida y características del autor en cuestión, realicen un breve esquema de los personajes y de sus circunstancias en el fragmento traducido en clase. De este modo, podrán evitar errores de interpretación derivados de una insuficiente contextualización del texto de prueba.

El material de referencia y los diccionarios son herramientas imprescindibles en la realización de una prueba, pero tienen ciertas limitaciones. Dado que los diccionarios empleados por los estudiantes a menudo no son lo suficientemente extensos, consideramos adecuado que el docente ponga a disposición de los alumnos diccionarios de mayor capacidad, diccionarios especializados o incluso un diccionario online, que puedan consultar durante la clase o durante la realización de una prueba en caso de que su material no ofrezca una solución para una determinada expresión. Este recurso de uso puntual puede paliar las limitaciones documentales a las que los alumnos se enfrentan en una situación de examen.

Especialmente en el caso de los alumnos germanoparlantes, hemos observado que una cuidadosa documentación de las explicaciones de clase ayuda a compensar las diferencias en el dominio del español con los alumnos hispanohablantes. Este fue el caso en la traducción de los conceptos 'acto, decorado, escena' del texto dramático, por ejemplo. Es necesario animar a los alumnos extranjeros a que se apoyen en esta estrategia en la mayor medida posible, quizá incluso compartiendo el 
material y los glosarios o presentándolos al docente regularmente para su revision.

Además de estas propuestas para mejorar el trabajo de clase y la evaluación continua, sería también deseable que el método de evaluación se enfocara desde otro punto de vista, más gratificante para los alumnos. Este sería el concepto de la 'evaluación positiva' tal como lo explica Christiane Nord. ${ }^{30}$ De este modo, se establecerían los objetivos que el alumno debe superar en una prueba concreta, o dicho de otro modo el horizonte de expectativas, y se puntuaría de manera positiva cada elemento superado.

Una futura línea de trabajo consistirá, por tanto, en aplicar el esquema de tipología y evaluación del error propuesto a un sistema de evaluación positiva específico para la corrección de cada prueba.

\section{REFERENCIAS BIBLIOGRÁFICAS}

\section{Libros y capítulos}

Balbuena TOReZANo, $\mathrm{M}^{\mathrm{a}}$ del Carmen, "La evaluación de traducciones al castellano de textos periodísticos alemanes realizadas por alumnos germanoparlantes", en VARELA SALINAS, Mª José (ed.), 2006, págs. 171-188.

\footnotetext{
${ }^{30}$ Nord, C. (2006:43): “...para fomentar la motivación de los alumnos y animarlos a pensar desde un punto de vista funcional, proponemos una evaluación positiva que destaque precisamente los logros en vez de los fracasos. Se trata de la diferencia entre el 'vaso medio lleno' y el 'vaso medio vacío'. Una evaluación positiva funciona de la siguiente manera: del total de los PT [problemas de traducción] de una determinada tarea, con su correspondiente peso expresado en puntos, restamos los que no fueron solucionados adecuadamente. Después sumamos los puntos de recompensa por la solución adecuada de unos PT no tratados anteriormente en clase. Podemos también 'bonificar' algunos puntos por un problema medio resuelto, donde se ve que el alumno ha reflexionado sobre una estrategia adecuada que luego no ha sabido desarrollar en su totalidad. El resultado representa la proporción de soluciones logradas. De manera que ya no constatamos que un alumno etiene un cincuenta por ciento de soluciones inadecuadas o de errores", sino que "ya ha solucionado adecuadamente el cincuenta por ciento de los PT»."
} 
BALBUENA tOREzANO, $\mathrm{M}^{a}$ del Carmen, "La traducción alemán-español de textos periodísticos realizada por alumnos hispanohablantes: principales errores traductologicos", en BALBUENA TOREZANO, $M^{3}$ del Carmen; GARCÍA CALDERON, Ángeles (eds.), 2007, págs. 381-389.

BAlbuena TORezano, $\mathrm{M}^{a}$ del Carmen; García Calderón, Ángeles (eds.), Traducción y mediación cultural. Reflexiones interdisciplinares, Granada, Atrio, 2007.

BALbUENA TOREZANO, $\mathrm{M}^{\mathrm{a}}$ del Carmen, "Texto literario y texto fílmico: una propuesta para la enseñanza de la traducción literaria alemán-español", en SILVIA ROISS, CARLOS FORTEA GIL, BELÉN SANTANA LÓPEZ En las vertientes de la traducción e interpretación del/al alemán, Berlín, Frank \& Timme, 2011, págs. 293-302.

DELISLE, Jean, "Définition, rédaction et utilité des objectifs d'apprentissage en enseignement de la traduction", en GARCÍA IZQUIERDO, Isabel; VERDEGAL, Joan (eds.), 1998, págs. 13-43.

ElENA GARCíA, Pilar, Aspectos teóricos y prácticos de la traducción (alemánespañol), Salamanca, Ediciones Universidad de Salamanca, 1990.

GARCIAA IZQUIERDO, Isabel; VERDEGAL, Joan (eds.), Los estudios de traducción: un reto didáctico, Castellón, Publicacions de la Universitat Jaume I, 1998.

HÖNIG, Hans G., "Konstruktives Übersetzen", en SNELL-HORNBY, Mary (ed.), Studien zur Translation (Volume 1), Tübingen, Stauffenburg Verlag, 1995.

HURTADO AlBIR, Amparo, "La enseñanza de la traducción directa 'general'. Objetivos de aprendizaje y metodología", en HURTADO ALBIR, Amparo (ed.), La enseñanza de la traducción, Castellón, Publicacions de la Universitat Jaume I, 1996.

Kussmaul, Paul, Training the Translator, Benjamins Translation Library (Volume 10), Ámsterdam/Filadelfia, John Benjamins Publishing Company, 1995.

MARTÍNEZ, Laura, “Orientación profesional en la enseñanza de la traducción literaria", en BALBUENA TOREZANO, $\mathrm{M}^{\mathrm{a}}$ del Carmen; GARCíA CALDERÓN, Ángeles (eds.), 2007.

NORD, Christiane, "El error en la traducción: categorías y evaluación", en HURT ADO ALBIR, Amparo (ed.), 1996, págs. 91-107.

NORD, Christiane, "El horizonte de expectativas en la evaluación funcional", en VARELA SALINAS, $\mathrm{M}^{\mathrm{a}}$ José (ed.), 2006, págs. 31-46.

RUIZ YEPES, Guadalupe, Teorías traductológicas y su incidencia sobre la evaluación de traductores, en VARELA SALINAS, $\mathrm{M}^{\text {a }}$ José (ed.), 2006, págs. 13-29.

SNELL-HORNBY, Mary, Translation studies: an integrated approach. Revised edition, Ámsterdam/Filadelfia, John Benjamins Publishing Company, 1995.

VARELA SALINAS, Ma José (ed.), La evaluación en los estudios de Traducción e Interpretación, Sevilla, Bienza, 2006.

Futhark 9 (2014)

Castillo, Análisis de las causas de errores, 35-64

ISSN 1886-9300 


\section{Legislación}

DECRETO 2549/1972 de 18 de agosto de 1972, por el que se crea, con carácter experimental, la Escuela Universitaria de Idiomas en la Universidad Autónoma de Barcelona (BOE del 22 de septiembre de 1972).

DECRETO 2293/1973, de 17 de agosto, por el que se regulan las Escuelas Universitarias (B.O.E. 231 de 26 de septiembre de 1973).

REAL DECRETO 2572/1979, de 14 de septiembre de 1979, por el que se transforma el actual Instituto de Idiomas, dependiente de la Universidad de Granada, en Escuela Universitaria de Traductores e Intérpretes, integrada en dicha Universidad (B.O.E. 269 de 9 de noviembre de 1979).

Manuales de clase

BALBUENA TOREZANO, $\mathrm{M}^{\mathrm{a}}$ del Carmen; COBOS, Ingrid, Introducción a la traducción no especializada (alemán-español), Córdoba, Ediciones Don Folio, 2011.

BALbUENA TOREZANO, $\mathrm{M}^{\mathrm{a}}$ del Carmen, Traducir literatura: presupuestos teóricos y prácticos para la traducción alemán-español de textos literarios, Córdoba, Ediciones Don Folio, 2011.

\section{Otros}

Guía de grado en traducción e interpretación, Universidad de Granada, en http://grados.ugr.es/traduccion/pages/infoacademica/guiagrado (fecha de consulta: abril de 2012)

Libro Blanco del Título de Grado en Traducción e Interpretación, Agencia Nacional de Evaluación de la Calidad y Acreditación, en http://www.aneca.es/var/media/150288/libroblanco_traduc_def.pdf (fecha de consulta: abril de 2012)

Guía docente de Traducción General alemán-español de la Licenciatura en Traducción e Interpretación de la Universidad de Córdoba, en http://www.uco.es/filosofiayletras/principal/normasdocumentos/documentos/guias/1112/traduccion/6477.pdf (fecha de consulta: 13-02-2012)

Guía docente de Traducción Literaria alemán-español de la Licenciatura en Traducción e Interpretación de la Universidad de Córdoba, en http://www.uco.es/filosofiayletras/principal/normasdocumentos/documentos/guias/1213/traduccion/6478.pdf (fecha de consulta: 13-02-2012)

Proyecto Gutenberg en alemán: http:/ / gutenberg.spiegel.de/ (fecha de consulta: 13-02-2012) 